\title{
REGIONAL OBSERVATION AND SENSORS
}

\author{
ABDELHAQ EL JAI, HOURIA HAMZAOUI \\ LAMPS—Systems Theory Laboratory \\ University of Perpignan, 52 avenue Paul Alduy, 66860 Perpignan Cedex, France \\ e-mail: $\{$ aej, houria.bergheul\}@univ-perp.fr
}

\begin{abstract}
The purpose of this short paper is to provide original results related to the choice of the number of sensors and their supports for general distributed parameter systems. We introduce the notion of extended sensors and we show that the observation error decreases when the support of a sensor is widened. We also show that the observation error decreases when the number of sensors increases.
\end{abstract}

Keywords: distributed parameter systems, sensors, observability, observation error.

\section{Preliminaries}

Many works on distributed parameter systems (DPSs) have been devoted to the observation problem (Curtain and Pritchard, 1978). It has often been studied independently of any geometric considerations, and most of the works were focused on the observation and reconstruction of the state in a certain observation space. The notion of sensors and actuators introduced in the 1980s by El Jai and Pritchard allows for a better description of measurements and actions, see (El Jai and Pritchard, 1988). Additionally, the study of observability and controllability can be considered with respect to the structure, number and location of sensors and actuators. For linear DPSs, observability and controllability are dual notions and most results on observability can be deduced from those on controllability by duality, see (Curtain and Pritchard, 1978; El Jai and Pritchard, 1988).

In the case of regional observability, the problem concerns the state observation in a subregion $\omega$ of $\Omega$ (Amouroux et al., 1994). It was shown that, in this regional case, the observation error is smaller than in the general observation case. Various motivating examples of regional observability are given in (Afifi et al., 2008; Amouroux et al., 1994; El Jai et al., 1995). In this paper we bring to light the link between the regional observation error and sensor structures (support and number). During the last decades very few papers have been devoted to this problem. Here we give interesting results which confirm and prove various simulation results like those given in (El Jai and El Yacoubi, 1993; El Jai and
Najem, 1984; El Jai, 1977) for actuators and in (Korbicz and Gawłowicz, 1990) for sensors.

The paper is organized as follows: First we provide results on regional observability for general systems, and we also consider the linear case. In the next section we define the notion of an extended sensor and its relation to the observation error. The last section is devoted to the number of sensors in connection with the observation error in the cases of internal, pointwise and boundary sensors.

Let $\Omega$ be a regular bounded open set of $\mathbb{R}^{n}$ with a smooth boundary $\partial \Omega, \omega$ a non-empty subset of $\Omega$, and let $[0, T]$ with $T>0$ be a time interval. We consider a distributed parameter system $(S)$ whose state is denoted by $z \in L^{2}(0, T ; Z)$, where $Z$ is the state space, considered generally as $L^{2}(\Omega)$. The state $z$ is assumed to satisfy convenient boundary conditions, but the initial state $z_{0}$ is supposed to be unknown. The usual distributed parameter models are governed by partial differential equations. In this paper most of the results are stated for abstract distributed parameter systems. A particular case of linear DPSs is developed in Section 1.3

An output function $y: Z \rightarrow \mathcal{O}=L^{2}(0, T ; Y)$ yields measurements and can be stated in the form

$$
y(t)=y\left(t, z, z_{0}, \Theta\right),
$$

emphasizing the fact that the output $y$ depends on an initial state $z_{0}$. It also depends on time, on the system dynamics and on a parameter $\Theta$ which defines the nature of the measurements. In the case of $q$ sensors, $\Theta$ is a vector of $2 q+1$ components which are $q$ sensor supports, $q$ spatial distributions of the sensors and the number of sensors. Usually, 
the output space is $Y=\mathbb{R}^{q}$. This will be made precise later.

Let $Q$ be the operator

$$
Q: z_{0} \in Z \rightarrow y \in \mathcal{O}
$$

which maps the state space into the observation space. The operator $Q$ depends on the output $y$ together with the system dynamics.

1.1. General definitions. Observability. We have the following observability definitions as stated in (Amouroux et al., 1994).

Definition 1. The system $(S)$ augmented with the output (1) is observable if the operator $Q$ is injective.

So when the system $(S)$ augmented with the output (1) is observable, a reconstruction of the state may be possible, and it depends on the dynamics and the output operators.

Regional observability. For regional observability we introduce the restriction mapping

$$
\chi_{\omega}: L^{2}(\Omega) \rightarrow L^{2}(\omega)
$$

defined by

$$
\chi_{\omega} z=\left.z\right|_{\omega}
$$

Definition 2. The system $(S)$ together with the output (1) is said to be regionally observable on $\omega$ or $\omega$-observable if the operator

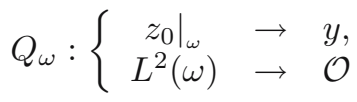

is injective. The state $\left.z_{0}\right|_{\omega}=\chi_{\omega} z_{0}$ holds for the restriction of $z_{0}$ to $\omega$. The state $z_{0}$ is said to be an $\omega$-observable state.

Remark 1. If the system is observable, then it is $\omega$ observable for any $\omega \subset \Omega$.

The above definition means that we are only interested in the state observation inside the subdomain $\omega$ but not in the whole domain $\Omega$. It is less restrictive than the previous definition (1). An $\omega$-observable state is a state which can be regionally reconstructed in the region $\omega$. Many characterization results and applications were developed in (El Jai et al., 1995; El Jai et al., 1995).

Sensors. Sensors are intermediaries between a system and its environment. They are characterized by the geometry of the supports, the location of the supports and the spatial distribution of the measurement on the support. We

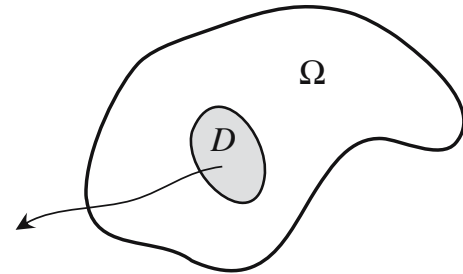

Fig. 1. General sensor structure.

can have many geometries for the supports (circular, rectangular, etc.) and many possibilities for their location. As in (El Jai and Pritchard, 1988), we define the sensor structure in a DPS as follows.

Definition 3. A sensor $\mathcal{C}$ is a couple $(D, f)$, where $D$ is the geometric support of the measurement and $f$ is the spatial distribution of the measurement on $D$. A sensor $\mathcal{C}$ is said to be zonal if $D$ is a nonempty subset of $\Omega$, thus $f \in L^{2}(D)$. It is said to be pointwise if $D=\{b\}$, thus $f=\delta_{b}$, where $\delta_{b}$ is the Dirac mass in $b$.

Some sensors have structures such that they allow the system to be observable or regionally observable. Let $\omega$ be a subregion of $\Omega, \omega \subset \Omega$.

Definition 4. A sensor $\mathcal{C}=(D, f)$ is said to be $\omega$ strategic if the observed system is $\omega$-observable.

Remark 2. It is clear that if a sensor is $\Omega$-strategic, then it is $\omega$-strategic.

When the system is $\omega$-observable, the state reconstruction may depend continuously or not on the output. This leads to different degrees of observability and regularity of the reconstructed state. For linear distributed systems, the state reconstruction can be done using various approaches. In (Amouroux et al., 1994), the state construction is based on pseudo-inverse techniques.

1.2. Regional observation error. The general observation problem in deciding whether the knowledge of the output together with the system dynamics makes the state reconstruction possible. When the system is observable, the state reconstruction leads necessarily to a reconstruction error, also called the observation error.

In the usual observation problem, we consider the error $E\left(z_{0}\right)$ defined by

$$
E\left(z_{0}\right)=\|y(t)-\widetilde{y}(t)\|_{L^{2}\left(0, T ; \mathbb{R}^{q}\right)}^{2},
$$

where $\widetilde{y}$ holds for the measurement and $y$ for the output. In our case, the observation error clearly depends on the target region $\omega$ where the state is to be observed together with the structure and number of sensors. This is why we introduce the following notation. 


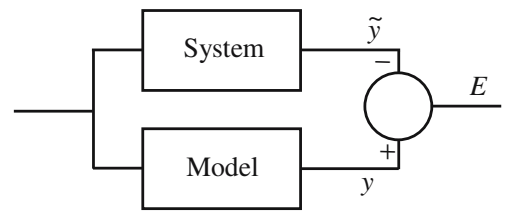

Fig. 2. Definition of the observation error. by

The set of regionally observable states in $\omega$ is denoted

$$
\mathcal{E}_{\omega}=\{\omega \text {-observable states }\} .
$$

The set $\mathcal{E}_{\Omega}$ stands for the set of observable states (in the whole domain $\Omega$ ). The associated observation error is then denoted by

$$
E_{\omega}: z_{0} \in \mathcal{E}_{\omega} \longrightarrow E_{\omega}\left(z_{0}\right) \in \mathbb{R} .
$$

The set of regionally observable states in $\omega$ when measurements are obtained by means of one zone sensor $\mathcal{C}$ is denoted by

$$
\mathcal{E}_{\omega}^{\mathcal{C}}=\{\omega \text {-observable states by means of the sensor } \mathcal{C}\} \text {. }
$$

The observation error associated with the set 9 is denoted by

$$
E_{\omega}(\cdot, \mathcal{C}): z_{0} \in \mathcal{E}_{\omega}^{\mathcal{C}} \longrightarrow E_{\omega}\left(z_{0}, \mathcal{C}\right) \in \mathbb{R} .
$$

When measurements are obtained by $k$ sensors $\left(\mathcal{C}_{i}\right)_{1 \leq i \leq k}$, we consider the set

$$
\begin{aligned}
\mathcal{E}_{\omega}^{\mathcal{C}_{\{1,2, \ldots, k\}}=} & \{\omega \text {-observable states } \\
& \text { by means of the sensors } \\
& \left.\mathcal{C}_{1}, \mathcal{C}_{2}, \ldots, \mathcal{C}_{k}\right\} .
\end{aligned}
$$

The associated observation error is then denoted by

$$
E_{\omega}\left(\cdot, \mathcal{C}_{1}, \mathcal{C}_{2}, \ldots, \mathcal{C}_{k}\right):\left\{\begin{array}{l}
\mathcal{E}_{\omega}^{\mathcal{C}_{\{1,2, \ldots, k\}}} \longrightarrow \mathbb{R} \\
z_{0} \longrightarrow E_{\omega}\left(z_{0}, \mathcal{C}_{1}, \mathcal{C}_{2}, \ldots, \mathcal{C}_{k}\right)
\end{array}\right.
$$

\section{Property 1.}

(i) For any nonempty region $\omega, \omega \subset \Omega$, we have

$$
\mathcal{E}_{\Omega} \subset \mathcal{E}_{\omega} .
$$

(ii) For any nonempty region $\omega$ and any sequence of zone sensors $\left(\mathcal{C}_{k}\right)_{1 \leq k \leq q}$, we have

$$
\mathcal{E}_{\omega}^{\mathcal{C}_{\ell}} \subset \mathcal{E}_{\omega}^{\mathcal{C}_{\{1,2, \ldots, k\}}} \subset \mathcal{E}_{\omega}
$$

for all $k, \ell, 1 \leq \ell \leq k$ and $1 \leq k \leq q$.
Proof. (i) If a state is observable in the whole $\Omega$, then it is observable in any subregion $\omega$ of $\Omega$, see Remark 1 Then $\mathcal{E}_{\Omega} \subset \mathcal{E}_{\omega}$.

(ii) Let $\left(\mathcal{C}_{k}\right)_{k}$ be a sequence of $q$ sensors. Obviously, for all $k, 1 \leq k \leq q, k$ sensors permit to observe more states than one sensor $\mathcal{C}_{\ell}$, and thus $\mathcal{E}_{\omega}^{\mathcal{C}_{\ell}} \subset \mathcal{E}_{\omega}^{\mathcal{C}_{\{1,2, \ldots, k\}}}$. On the other hand, it is clear that any finite sequence of sensors does not allow for the observability (or $\omega$-observability) of all the observable states, and therefore $\mathcal{E}_{\omega}^{\mathcal{C}_{\{1,2, \ldots, k\}}} \subset \mathcal{E}_{\omega}$.

\section{Remark 3.}

(i) In the case of pointwise sensors, in the above notation the sensor $\mathcal{C}$ is replaced by the location $b$. The sets given by (9) and (11) become respectively $\mathcal{E}_{\omega}^{b}$ and $\mathcal{E}_{\omega}^{b_{1}, b_{2}, \ldots, b_{k}}$.

(ii) In the case of boundary zone (respectively pointwise) sensors, the notation remains the same except that the sensor supports (resp. locations) are subsets of the boundary $\partial \Omega$ of the domain $\Omega$.

The reconstruction method is based on finding a state $z_{0}^{\star}$ which realizes

$$
E\left(z_{0}^{\star}\right)=\inf _{z_{0}} E\left(z_{0}\right)
$$

Note that the minimum $E\left(z_{0}^{\star}\right)$ depends on how the measurements have been considered, i.e., the number and structure of sensors. Thus we write

$$
E\left(z_{0}^{\star}\right)=E\left(z_{0}^{\star}, \mathcal{C}\right)
$$

for the observation error depending on the sensor $\mathcal{C}$. Additionally, we assume that the mapping $\mathcal{C} \longrightarrow E\left(z_{0}^{\star}, \mathcal{C}\right)$ is continuous. This means that if the sensor parameters are slightly modified, then the observation error changes continuously. In the case of $k$ sensors, i.e., a multi-output situation, it will be denoted by

$$
E\left(z_{0}^{\star}\right)=E\left(z_{0}^{\star}, \mathcal{C}_{1}, \mathcal{C}_{2}, \ldots, \mathcal{C}_{k}\right) .
$$

When the sensors are pointwise, we write

$$
E\left(z_{0}^{\star}\right)=E\left(z_{0}^{\star}, b_{1}, b_{2}, \ldots, b_{k}\right) .
$$

The notation remains similar in the boundary case except that the supports and locations of sensors are subsets of $\partial \Omega$.

In what follows we consider the observation error function defined by (8), (10) and (12) depending on the case considered. We assume that the functions $E, E(\cdot, \mathcal{C})$ and $E\left(\cdot, \mathcal{C}_{1}, \mathcal{C}_{2}, \ldots, \mathcal{C}_{k}\right)$ are convex with respect to the initial state $z_{0}$. Therefore we immediately have the following important result.

Proposition 1. Let $\omega$ be a given region of $\Omega$. Suppose that the system $(S)$ with the output (1) is regionally observable on $\omega$. Then the observation error in $\omega$ is lower than that in the whole domain $\Omega$. 
Proof. Consider the sets $\mathcal{E}_{\omega}$ and $\mathcal{E}_{\Omega}$. Since $\omega \subset \Omega$, by Remark 2, any observable state in $\Omega$ is observable in $\omega$ and then $\mathcal{E}_{\Omega} \subset \mathcal{E}_{\omega}$, because observability implies regional $\omega$-observability for any $\omega \subset \Omega$. Therefore,

$$
\inf _{z_{0} \in \mathcal{E}_{\omega}} E\left(z_{0}\right) \leq \inf _{z_{0} \in \mathcal{E}_{\Omega}} E\left(z_{0}\right) .
$$

\section{Remark 4.}

(i) As a generalization of the above result, we can demonstrate that if $\omega_{1}$ and $\omega_{2}$ are two subdomains of $\Omega$ such that $\omega_{1} \subset \omega_{2}$, then

$$
\inf _{z_{0} \in \mathcal{E}_{\omega_{1}}} E\left(z_{0}\right) \leq \inf _{z_{0} \in \mathcal{E}_{\omega_{2}}} E\left(z_{0}\right)
$$

The proof is similar and proceeds by replacing $\omega$ by $\omega_{1}$ and $\Omega$ by $\omega_{2}$.

(ii) The above result means that the smaller the region $\omega$ where the state is to be observed, the lower the corresponding observation error. The result remains true even if the region considered is on the boundary $\partial \Omega$, with additional regularity assumptions.

The regional observation error on $\omega$ is assumed to be convex, and we write

$$
E_{\omega}\left(z_{0}^{\star}\right)=\inf _{z_{0} \in \mathcal{E}_{\omega}} E\left(z_{0}\right)
$$

and $E_{\Omega}\left(z_{0}^{*}\right)=E\left(z_{0}^{\star}\right)$ holds for $\inf _{z_{0} \in \mathcal{E}_{\Omega}} E\left(z_{0}\right)$.

1.3. Linear case. In order to make the results precise, consider the particular case where the system $(S)$ is linear and given by the following abstract state equation:

$$
\left\{\begin{array}{l}
\dot{z}(t)=A z(t)+B u(t), \quad 0 \leq t \leq T, \\
z(0)=z_{0}
\end{array}\right.
$$

with the output function

$$
y(t)=C z(t), \quad 0 \leq t \leq T,
$$

where $C$ is a linear operator from $Z$ into $L^{2}\left(0, T ; \mathbb{R}^{q}\right)$. Additionally, we assume that the operator $A$ generates a strongly continuous semigroup $(S(t))_{t \geq 0}$ on the state space $Z=L^{2}(\Omega)$ and is self-adjoint with compact resolvent. We have $u \in L^{2}\left(0, T ; \mathbb{R}^{p}\right)$ and $y \in L^{2}\left(0, T ; \mathbb{R}^{q}\right)$.

When the system (22) is autonomous, (23) yields

$$
y(t)=C S(t) z_{0}=K(t) z_{0}
$$

where $K$ is an operator from $L^{2}(\Omega)$ into $L^{2}(0, T ; Z)$. The adjoint $K^{*}$ is given by

$$
K^{*} z^{*}=\int_{0}^{T} S^{*}(T-s) C^{*} z^{*} \mathrm{~d} s .
$$

In the linear case, regional observability can be characterized by the following property. Consider a given region $\omega$ and the restriction function

$$
\chi_{\omega}: z \in L^{2}(\Omega) \longrightarrow \chi_{\omega} z=\left.z\right|_{\omega} \in L^{2}(\omega)
$$

whose adjoint $\chi_{\omega}^{*}: L^{2}(\omega) \longrightarrow L^{2}(\Omega)$ is given by

$$
\left(\chi_{\omega}^{*} z\right)(x)= \begin{cases}z(x), & x \in \omega, \\ 0, & x \in \Omega \backslash \omega,\end{cases}
$$

where $x$ stands for the space variable depending on the dimension of the operator domain.

\section{Property 2.}

(i) The system (22) with the output (23) is exactly $\omega$ observable if

$$
\operatorname{Im} \chi_{\omega} K^{*}=L^{2}(\omega) .
$$

(ii) The system (22) with the output (23) is weakly $\omega$ observable if

$$
\overline{\operatorname{Im} \chi_{\omega} K^{*}}=L^{2}(\omega) .
$$

In the linear case, the reconstruction operator $Q_{\omega}$ defined in (5) can be expressed by a pseudo-inverse which exists in the case of weak $\omega$-observability and which is continuous in the case of exact $\omega$-observability, see (El Jai and Pritchard, 1988). Let $K_{\omega}=\chi_{\omega} K$. Then it has been shown that if $K_{\omega}^{*} K_{\omega}$ is positive (respectively positive definite), then the system (22) augmented with the output (23) is weakly observable (resp. exactly observable), and the state $z_{0}$ is given by

$$
z_{0}=K_{\omega}^{\dagger} y
$$

where $K_{\omega}^{\dagger}$ is the pseudo-inverse operator of $K_{\omega}$ given by $K_{\omega}^{\dagger}=\left(K_{\omega}^{*} K_{\omega}\right)^{-1} K_{\omega}^{*}$.

From the above, we deduce the following characterization.

Proposition 2. The system (22) with the output (23) is exactly $\omega$-observable if there exists $\nu>0$ such that, for all $z_{0} \in Z=L^{2}(\Omega)$,

$$
\left\|\chi_{\omega} z_{0}\right\|_{L^{2}(\omega)} \leq \nu\left\|K \chi_{\omega}^{*} z_{0}\right\|_{L^{2}(0, T ; Z)} .
$$

Remark 5. The characterization (29) is more general than that in which $\omega=\Omega$. For this case we can use the result given in (Curtain and Pritchard, 1978).

Consider a sequence of sensors $\left(D_{i}, f_{i}\right)_{1<i<q}$. It is possible to choose their structure (supports or space distributions) so as to make them $\omega$-strategic. More precisely, we have the following results, shown in (El Jai and Pritchard, 1988).

Proposition 3. For the linear system (22) with the output (23), if the supports $\left(D_{i}\right)_{1 \leq i \leq q}$ of the sensors are given, there always exist distributions $\left(f_{i}\right)_{1 \leq i \leq q}$ such that the sensors $\left(D_{i}, f_{i}\right)_{1 \leq i \leq q}$ are $\omega$-strategic. 
Moreover, we show that for all $i, 1 \leq i \leq q$, the spatial distribution $f_{i} \in L^{2}\left(D_{i}\right)$.

Proposition 4. For the linear system (22) with the output (23), if the spatial distributions $\left(f_{i}\right)_{1 \leq i \leq q}$ are given, there always exist locations for the supports $\left(D_{i}\right)_{1 \leq i \leq q}$ with $D_{i} \subset \Omega$ such that the sensors $\left(D_{i}, f_{i}\right)_{1 \leq i \leq q}$ are $\omega$ strategic.

These results are related to the existence of an adequate location or space distribution for a given type of measurement that makes the sensors strategic. Recall the so-called rank characterization of strategic sensors. With the hypothesis considered, the operator $A$ has a complete set of eigenfunctions $\left(\varphi_{n j}\right)$ associated with the eigenvalues $\left(\lambda_{n}\right)$ where $\lambda_{n}$ is of multiplicity $r_{n}$. The determination of the eigenfunctions depends on the form of the operator domain together with its dimension $n, \Omega \subset \mathbb{R}^{n}$.

Proposition 5. The zone sensors $\left(D_{i}, f_{i}\right)_{1 \leq i \leq q}$ are $\omega$ strategic if and only if

$$
\begin{aligned}
& \text { 1. } q \geq \sup _{n} r_{n}, \\
& \text { 2. } \operatorname{rank}\left(G_{n} \gamma_{\omega}^{n}\right)=r_{n}, \forall n \text {, }
\end{aligned}
$$

where $G_{n}$ is the $q \times r_{n}$ matrix defined by

$$
\left(G_{n}\right)_{i j}=\left\langle f_{i}, \varphi_{n j}\right\rangle_{L^{2}\left(D_{i}\right)}
$$

for $i=1, \ldots, q$ and $j=1, \ldots, r_{n}$, and

$$
\gamma_{\omega}^{n}=\left[\begin{array}{llll}
\gamma^{n_{1}} & \gamma^{n_{2}} & \ldots & \gamma^{n_{r_{n}}}
\end{array}\right]^{\mathrm{tr}}
$$

with $\gamma^{n_{i}}=\left(\gamma_{n_{i} j_{k}}\right), j=1,2, \ldots$, and $k=1, \ldots, r_{j}$,

$$
\gamma_{n_{i} j_{k}}=\int_{\omega} \varphi_{n_{i}} \varphi_{j_{k}} \mathrm{~d} x .
$$

The above result provides a rank condition which links the structure, location and number of the sensors to their possibility of making the system $\omega$-observable. The above rank condition was extended to unbounded cases when the sensors are pointwise or located on the boundary (Afifi et al., 2008).

Remark 6. The first condition of Proposition 5 needs the sensors number to be greater than $\sup _{n} r_{n}$. In (El Jai and El Yacoubi, 1993) it was shown that, using the results on the eigenvalues multiplicity established in (Micheletti, 1976), one sensor may be sufficient to ensure system observability assuming that the space domain $\Omega$ is slightly modified to a domain $\Omega^{*}$ such that $d\left(\Omega, \Omega^{*}\right) \leq \varepsilon$, where $d$ is the Hausdorff metric and $\varepsilon$ is sufficiently small.

\section{Sensors and observation error}

In this section we consider a distributed parameter system $(S)$ and we study the observation error with respect to the sensor structure. The developed approach is inspired by the regional analysis of DPSs, i.e., when the objective is only considered in a subdomain of $\Omega$.

If the observation is obtained by means of one zone sensor $\mathcal{C}=(D, f)$, we assume that the output of the system $(S)$ is given by

$$
y=y_{\mathcal{C}}=\langle f, z\rangle_{L^{2}(D)}=\int_{D} f z \mathrm{~d} x,
$$

where $z$ stands for the state of the system $(S)$. In the case of $q$ sensors, the above output is a vector function with $q$ components, the $i$-th component being related to the $i$-th sensor.

2.1. Sensor extension. Let $\omega$ be a subregion of $\Omega$. We consider a zone sensor $\mathcal{C}=(D, f), D \subset \Omega$ and $f \in L^{2}(D)$. By analogy to what was considered for actuators in (El Jai and Hamzaoui, 2008), we introduce the following definition.

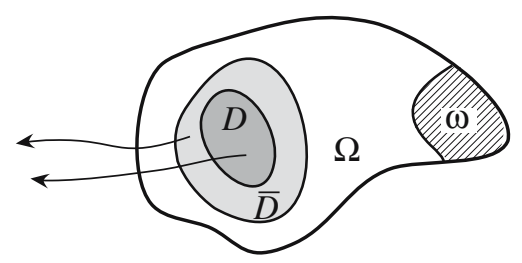

Fig. 3. Definition of an extended sensor.

Definition 5. A sensor $\overline{\mathcal{C}}=(\bar{D}, \bar{f})$ is said to be an extended sensor of $\mathcal{C}=(D, f)$, which is denoted by $\overline{\mathcal{C}} \geq$ $\mathcal{C}$, if the following conditions hold:

1. $\bar{D} \supset D$.

2. The function $\bar{f}$ is defined by

$$
\bar{f}= \begin{cases}f & \text { on } D, \\ h & \text { on } \bar{D} \backslash D,\end{cases}
$$

where $h$ is an arbitrary function such that $h \in L^{2}(\bar{D} \backslash D)$.

When a sensor $\overline{\mathcal{C}}$ extends a sensor $\mathcal{C}$, the set $\mathcal{E}_{\omega}^{\overline{\mathcal{C}}}$ of states observable by means of $\overline{\mathcal{C}}$ includes the set $\mathcal{E}_{\omega}^{\mathcal{C}}$ of states observable by means of $\mathcal{C}$, cf. Fig. 4

Remark 7. The above definition of extended sensors may also be considered by replacing the first condition by

$$
\operatorname{meas}(D) \leq \operatorname{meas}(\bar{D})
$$

where meas $(D)$ stands for the measure (area) of the support $D$. 


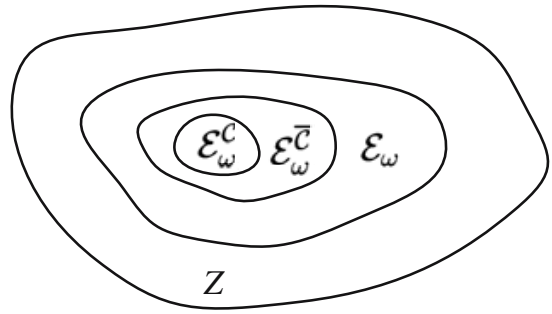

Fig. 4. Sets of observable states.

Proposition 6. If a system $(S)$ with the output $(32)$ is observed by means of a sensor $\mathcal{C}=(D, f)$, then the output $y_{\mathcal{C}}$ associated with $\mathcal{C}$ is equal to the output $y_{\overline{\mathcal{C}}}$ associated with the extended sensor $\overline{\mathcal{C}}=(\bar{D}, \bar{f})$ with $h=0$.

Proof. Suppose that the system (22) is observed by means of the extended sensor $\overline{\mathcal{C}}=(\bar{D}, \bar{f})$. Then

$$
\begin{aligned}
y_{\overline{\mathcal{C}}} & =\int_{\bar{D}} \bar{f}(x) z(x) \mathrm{d} x \\
& =\int_{D} \bar{f}(x) z(x) \mathrm{d} x+\int_{\bar{D} \backslash D} \bar{f}(x) z(x) \mathrm{d} x \\
& =\int_{D} f(x) z(x) \mathrm{d} x+\int_{\bar{D} \backslash D} h(x) z(x) \mathrm{d} x .
\end{aligned}
$$

As $h=0$ on $\bar{D} \backslash D$, we get

$$
y_{\overline{\mathcal{C}}}(t)=\int_{D} f(x) z(x) \mathrm{d} x=y_{\mathcal{C}}(t) .
$$

Finally, the output (23) is not modified when the system (22) is observed by means of a convenient extended sensor.

Corollary 1. If the sensor $\mathcal{C}$ is strategic, then so is the extended sensor $\overline{\mathcal{C}}$.

Remark 8. The previous result does not always hold if the extended sensor is defined by means of the measure extension in Remark 7

2.2. Observation error and sensor extension. Let $\omega$ be a given subregion of $\Omega$. The notation for the observation error $E_{\omega}\left(z_{0}^{*}, \mathcal{C}\right)$ given by (16) means that the observation error also depends on the sensor $\mathcal{C}$. Consider the set $\mathcal{E}_{\omega}^{\mathcal{C}}$ defined in (9) and the set $\mathcal{E}_{\omega}^{\overline{\mathcal{C}}}$ associated with the extended sensor $\overline{\mathcal{C}}=(\bar{D}, \bar{f})$.

Proposition 7. Assume that the sensors $\mathcal{C}=(D, f)$ and $\overline{\mathcal{C}}=(\bar{D}, \bar{f})$ are $\omega$-strategic. If the sensor $\overline{\mathcal{C}}$ extends the sensor $\mathcal{C}$, then

$$
E_{\omega}\left(z_{0}^{\star}, \overline{\mathcal{C}}\right) \leq E_{\omega}\left(z_{0}^{\star}, \mathcal{C}\right),
$$

where $E_{\omega}\left(z_{0}^{\star}, \overline{\mathcal{C}}\right)$ is defined as in (16) with the sensor $\overline{\mathcal{C}}$.
Proof. Since the observation error function $E_{\omega}$ is convex, $\inf _{z_{0}} E_{\omega}\left(z_{0}\right)=E_{\omega}\left(z_{0}^{*}\right)$ exists. The set $\mathcal{E}_{\omega}$ of $\omega$ observable states is a vector subspace and therefore it is closed. Thus $z_{0}^{\star} \in \mathcal{E}_{\omega}$. The state $z_{0}^{\star}$ is $\omega$-observable using the sensor $\mathcal{C}$, which implies that it is $\omega$-observable using the extended sensor $\overline{\mathcal{C}}$ (by Proposition 6 ). This yields $\mathcal{E}_{\omega}^{\mathcal{C}} \subset \mathcal{E}_{\omega}^{\overline{\mathcal{C}}}$. Therefore,

$$
E_{\omega}\left(z_{0}^{\star}, \overline{\mathcal{C}}\right) \leq E_{\omega}\left(z_{0}^{\star}, \mathcal{C}\right) .
$$

This result means that the observation error can be reduced only by extending the support of the measurements. Consider now the case where the measurements are obtained by means of a sequence of sensors $\left(\mathcal{C}_{i}\right)_{1 \leq i \leq q}=$ $\left(D_{i}, f_{i}\right)_{1 \leq i \leq q}$

Corollary 2. Let $\left(\mathcal{C}_{k}\right)_{k>1}$ be a sequence of $\omega$-strategic zone sensors. If for all $k, 1 \leq k \leq q$, the sensor $\mathcal{C}_{k+1}=$ $\left(D_{k+1}, f_{k+1}\right)$ extends the sensor $\mathcal{C}_{k}=\left(D_{k}, f_{k}\right)$, then we have

$$
E_{\omega}\left(z_{0}^{\star}, \mathcal{C}_{k+1}\right) \leq E_{\omega}\left(z_{0}^{\star}, \mathcal{C}_{k}\right), \quad \forall k \geq 1 .
$$

The proof can be easily deduced from Proposition 7

\section{Number of sensors}

In the previous section we have shown that it is possible to reduce the observation error only by extending the support of the measurements. In this section we will see that the number of sensors has the same effect on the observation error.

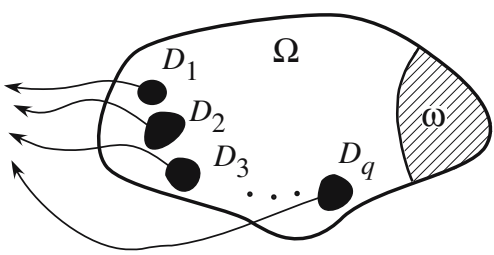

Fig. 5. Zone $\omega$-strategic sensors.

3.1. Number of sensors and the observation error. Consider a distributed system $(S)$ augmented with an output given by means of $q$ zone $\omega$-strategic sensors $\left(\mathcal{C}_{i}\right)_{1 \leq i \leq q}=\left(D_{i}, f_{i}\right)_{1 \leq i \leq q}$ such that $D_{i} \subset \Omega$ and $f_{i} \in$ $L^{2}\left(D_{i}\right)$ for $i=1, \ldots, q$. Let $E_{\omega}\left(z_{0}^{\star}\right)$ be the observation error defined in (21). We also assume that if, for some $i$ and $j, 1 \leq i, j \leq q$, the sensor supports are such that $D_{i} \cap D_{j} \neq \emptyset$, then $f_{i}=f_{j}$ on $D_{i} \cap D_{j}$. Thus we have the following important result.

Proposition 8. Assume that, for any $\ell, 1 \leq \ell \leq q$, the system is $\omega$-observable by means of $\ell$ sensors. Then the observation error decreases when the sensors number increases. 
Proof. Let the maximum number of sensors be $q$. Assume first that the system is observed by means of one $\omega$-strategic sensor $\mathcal{C}_{1}=\left(D_{1}, f_{1}\right)$.

If we consider a second $\omega$-strategic sensor $\mathcal{C}_{2}=$ $\left(D_{2}, f_{2}\right)$, then the system is observed by means of two $\omega$-strategic sensors. Now consider the two sensors $\mathcal{C}$ and $\overline{\mathcal{C}}$ as follows:

(i) $\mathcal{C}=(D, f)$ such that

$$
D=D_{1} \text { and } f=f_{1},
$$

(ii) $\overline{\mathcal{C}}=(\bar{D}, \bar{f})$ such that

$$
\bar{D}=D_{1} \cup D_{2} \quad \text { and } \quad \bar{f}= \begin{cases}f_{1} & \text { on } D_{1}, \\ f_{2} & \text { on } D_{2} .\end{cases}
$$

The sensors $\mathcal{C}$ and $\overline{\mathcal{C}}$ are $\omega$-strategic and, obviously, $\overline{\mathcal{C}}$ extends $\mathcal{C}$. Then, by Proposition (7), we have

$$
E_{\omega}\left(z_{0}^{\star}, \overline{\mathcal{C}}\right) \leq E_{\omega}\left(z_{0}^{\star}, \mathcal{C}\right)
$$

or, equivalently,

$$
E_{\omega}\left(z_{0}^{\star}, \mathcal{C}_{1}, \mathcal{C}_{2}\right) \leq E_{\omega}\left(z_{0}^{\star}, \mathcal{C}_{1}\right) .
$$

Suppose now that the system is observed by means of $k$ zone sensors $\left(\mathcal{C}_{i}=\left(D_{i}, f_{i}\right)\right)_{1 \leq i \leq k}$ assumed to be $\omega$ strategic and such that

$$
\begin{aligned}
E_{\omega}\left(z_{0}^{*}, \mathcal{C}_{1}, \mathcal{C}_{2}, \ldots, \mathcal{C}_{k}\right) & \leq \ldots \\
& \leq E_{\omega}\left(z_{0}^{*}, \mathcal{C}_{1}, \mathcal{C}_{2}\right) \\
& \leq E_{\omega}\left(z_{0}^{*}, \mathcal{C}_{1}\right) .
\end{aligned}
$$

Consider an additional sensor $\mathcal{C}_{k+1}=\left(D_{k+1}, f_{k+1}\right)$ and the following two sensors:

(i) $\mathcal{C}=(D, f)$ such that

$$
D=\bigcup_{i=1}^{k} D_{i} \text { and } f=\left\{\begin{array}{cc}
f_{1} & \text { on } D_{1}, \\
f_{2} & \text { on } D_{2}, \\
\vdots & \\
f_{k} & \text { on } D_{k},
\end{array}\right.
$$

(ii) $\overline{\mathcal{C}}=(\bar{D}, \bar{f})$ such that

$$
\bar{D}=\bigcup_{i=1}^{k+1} D_{i} \text { and } \bar{f}=\left\{\begin{array}{cl}
f_{1} & \text { on } D_{1}, \\
f_{2} & \text { on } D_{2}, \\
\vdots & \\
f_{k+1} & \text { on } D_{k+1}
\end{array}\right.
$$

Thus sensors $\mathcal{C}$ and $\overline{\mathcal{C}}$ are $\omega$-strategic and $\overline{\mathcal{C}}$ extends $\mathcal{C}$. By Proposition (7) we have

$$
E_{\omega}\left(z_{0}^{*}, \mathcal{C}_{1}, \mathcal{C}_{2}, \ldots, \mathcal{C}_{k+1}\right) \leq E_{\omega}\left(z_{0}^{*}, \mathcal{C}_{1}, \mathcal{C}_{2}, \ldots, \mathcal{C}_{k}\right)
$$

Finally,

$$
\begin{aligned}
E_{\omega}\left(z_{0}^{*}, \mathcal{C}_{1}, \mathcal{C}_{2}, \ldots, \mathcal{C}_{k+1}\right) & \leq E_{\omega}\left(z_{0}^{*}, \mathcal{C}_{1}, \mathcal{C}_{2}, \ldots, \mathcal{C}_{k}\right) \\
& \leq \ldots \\
& \leq E_{\omega}\left(z_{0}^{*}, \mathcal{C}_{1}, \mathcal{C}_{2}\right) \\
& \leq E_{\omega}\left(z_{0}^{*}, \mathcal{C}_{1}\right)
\end{aligned}
$$

The inequality (45) shows that the observation error obtained by means of $k+1$ sensors is lower than that obtained with $k$ sensors, and so on, and it is lower than that obtained by one sensor.

3.2. Active sensors statement. We consider a distributed parameter system $(S)$ augmented with an output expressed by means of $\omega$-strategic zone sensors. We assume that the maximum number of sensors is equal to $q$. Some of the sensors can be active and then the others are inactive. We also assume that if the sensor supports are such that $D_{i} \cap D_{j} \neq \emptyset$, then $f_{i}=f_{j}$ in $D_{i} \cap D_{j}$.

We consider the parameter vector $x=\left(x_{1}, \ldots, x_{q}\right)$ given by

$$
x_{i}= \begin{cases}1 & \text { if the } i \text {-th sensor is active } \\ 0 & \text { otherwise }\end{cases}
$$

and we define the observation error function in the following form:

$$
E_{\omega}^{q}\left(z_{0}^{\star}\right)=\sum_{i=1}^{q} x_{i} E_{\omega}\left(z_{0}^{\star}, \mathcal{C}_{i}\right),
$$

where $E_{\omega}\left(z_{0}^{\star}, \mathcal{C}_{i}\right)$ is the regional observation error due to the sensor $\mathcal{C}_{i}$ as defined in (16). When $k$ sensors are active for all $1 \leq k \leq q$, the parameter $x$ satisfies

$$
\sum_{i=1}^{q} x_{i}=k
$$

and the error 47) is denoted by $E_{\omega}^{k}\left(z_{0}^{\star}\right)$.

Now we are interested by the evolution of the observation error 47) with respect to the number of active sensors.

Remark 9. It is clear that, for $i \neq j$, the set of $\omega$ observable states by means of a sensor $\mathcal{C}_{i}$ may be different from the set of $\omega$-observable states by means of a sensor $\mathcal{C}_{j}$, see Fig. 6

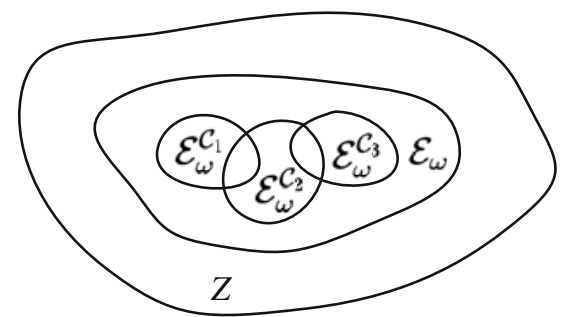

Fig. 6. Different sets of $\omega$-observable states.

Proposition 9. When the number of active sensors increases, the observation error (47) decreases. 
Proof. In much the same way as in the proof of Proposition 8 , we can show that, by successively activating additional sensors, the observation error decreases. At Step $k$ we activate $k$ sensors and at Step $(k+1)$ we activate $k+1$ sensors. When $k$ sensors are active, the parameter $x$ satisfies

$$
\sum_{i=1}^{q} x_{i}=k,
$$

and when $k+1$ sensors are active, we have

$$
\sum_{i=1}^{q} x_{i}=k+1 .
$$

Then it is clear that, for all $k$,

$$
E_{\omega}\left(z_{0}^{\star}, \mathcal{C}_{1}, \mathcal{C}_{2}, \ldots, \mathcal{C}_{k+1}\right) \leq E_{\omega}\left(z_{0}^{\star}, \mathcal{C}_{1}, \mathcal{C}_{2}, \ldots, \mathcal{C}_{k}\right),
$$

and therefore

$$
E_{\omega}^{k+1}\left(z_{0}^{\star}\right) \leq E_{\omega}^{k}\left(z_{0}^{\star}\right)
$$

\section{Remark 10.}

- The above result means that for reducing the observation error one have to activate all the sensors (whose maximum number is $q$ ).

- The previous proposition is general but does not take into account the case where the number of active sensors increases by activating different sensors from the first ones. Obviously, there exists a combination which can lead to the smallest error. This will depend on the system considered and can be found using simulation tests.

- The previous result is also true in the case of pointwise and boundary active sensors.

- All the previous results can be extended to the case of a regional boundary observability problem, i.e., when the region $\omega$ is a part of the boundary $\partial \Omega$.

3.3. Pointwise sensors. In this section we consider the problem of the observation error in the case of pointwise sensors assumed to be $\omega$-strategic, and we study the relation between zone and pointwise sensors. Let us consider the pointwise sensor $\mathcal{C}_{b}=\left(b, \delta_{b}\right)$ as given in Definition 3 and denote by $E_{\omega}\left(z_{0}^{*}, b\right)$ the associated observation error. In a complete metric space, the pointwise sensor location $b$ satisfies

$$
\{b\}=\bigcap_{i \geq 1} D_{i},
$$

where the closed sets $\left(D_{i}\right)_{i \geq 1}$ can be considered as the supports of zone sensors $\left(\mathcal{C}_{i}\right)_{i \geq 1}$ satisfying, for each $i$,

$$
\mathcal{C}_{i+1} \leq \mathcal{C}_{i}
$$

This means that the sensor $\mathcal{C}_{i}$ extends the sensor $\mathcal{C}_{i+1}$, for all each $i$. The sensor supports $\left(D_{i}\right)$ are spatially shrinking and converging to $b$ as $i \rightarrow \infty$. The sensors are defined by couples $\mathcal{C}_{i}=\left(D_{i}, f_{i}\right)$, cf. Definition 3 Additionally, a hypothesis which is consistent with physical consideration is that a pointwise sensor is considered as a zone sensor with a very small support. Thus we assume the zone sensors spatial distributions $\left(f_{i}\right)$ to be such that $\lim _{k \rightarrow+\infty} \mathcal{C}_{k}=\mathcal{C}_{b}$. Mathematically speaking, it can be stated by considering the Hausdorff distance on a convenient topological space, but this is not the purpose of the paper.

Proposition 10. Let $E_{\omega}\left(z_{0}^{*}, b\right)$ denote the observation er ror related to the pointwise sensor $\left(b, \delta_{b}\right)$ and $E_{\omega}\left(z_{0}^{\star}, \mathcal{C}_{k}\right)$ denote the observation error related to the zone sensors $\mathcal{C}_{k}$ assumed to satisfy (51). Then we have

$$
E_{\omega}\left(z_{0}^{*}, b\right)=\lim _{k \rightarrow+\infty} E_{\omega}\left(z_{0}^{\star}, \mathcal{C}_{k}\right) .
$$

Proof. Since the sensor $\mathcal{C}_{k}$ extends the sensor $\mathcal{C}_{k+1}$, for each $k \geq 1$, from Proposition 7 we have

$$
E_{\omega}\left(z_{0}^{\star}, \mathcal{C}_{k}\right) \leq E_{\omega}\left(z_{0}^{\star}, \mathcal{C}_{k+1}\right), \quad \forall k \geq 1
$$

Moreover, for each $k, b \in D_{k+1}$ the support of the sensor $\mathcal{C}_{k+1}$, and thus

$$
0 \leq E_{\omega}\left(z_{0}^{\star}, b\right) \leq E_{\omega}\left(z_{0}^{\star}, \mathcal{C}_{k}\right), \quad \forall k \geq 1 .
$$

We have $D_{k} \rightarrow\{b\}$ as $k \rightarrow \infty$, and using the assumption that $C_{k} \rightarrow C_{b}$ as $k \rightarrow \infty$ and the continuity of $\mathcal{C} \longrightarrow$ $E_{\omega}\left(z_{0}^{\star}, \mathcal{C}\right)$, we obtain

$$
E_{\omega}\left(z_{0}^{\star}, b\right)=\lim _{k \rightarrow+\infty} E_{\omega}\left(z_{0}^{\star}, \mathcal{C}_{k}\right) .
$$

The observation error related to a pointwise sensor can be considered as the limit of the observation errors of shrinking zone sensors. This also shows that the observation error related to the pointwise sensor can be estimated by considering a convenient zone sensor and the associated observation error. Thus, with Proposition 7] we have the following result.

Proposition 11. Assume that there exist q pointwise sensors located at points $b_{1}, b_{2}, \ldots, b_{q}$, and denote by $E_{\omega}\left(z_{0}^{\star}, b_{1}, b_{2}, \ldots, b_{k}\right)$ the observation error when the system is observed by $k$ pointwise sensors. Then we have

$$
E_{\omega}\left(z_{0}^{\star}, b_{1}, b_{2}, \ldots, b_{\ell}\right) \leq E_{\omega}\left(z_{0}^{\star}, b_{1}, b_{2}, \ldots, b_{k}\right)
$$

for all $k, \ell, 1 \leq k \leq \ell \leq q$.

This result extends naturally the previous results on zone sensors showing that, even in the pointwise case, the observation error decreases when the number of sensors increases. 


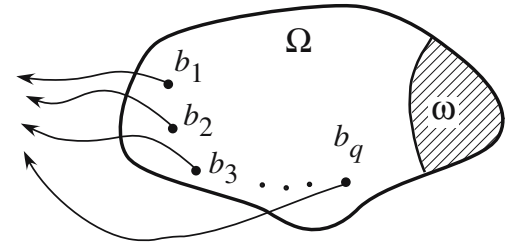

Fig. 7. Illustration of Proposition 11.

Remark 11. Combining the results of Propositions 8 and 11. we see that an observation error function can be reduced by increasing the total amount of space used for observing the system, both in the pointwise and zone cases.

3.4. Boundary sensors. The previous results can be extended to the case where the sensors are located on a regular boundary $\partial \Omega$ of the domain $\Omega$. As shown in Fig. 8 , the sensors may be zonal or pointwise.

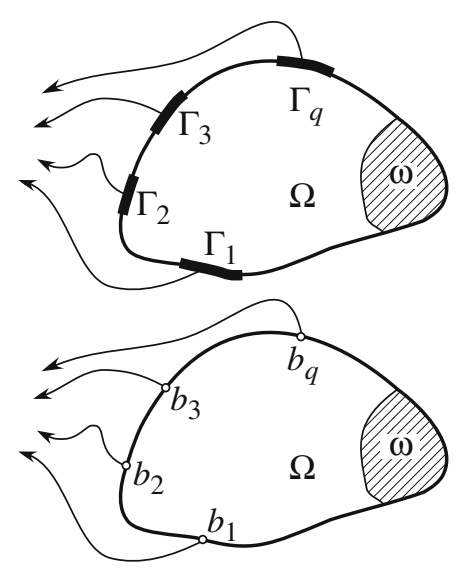

Fig. 8. Boundary zone and boundary pointwise sensors.

In the case of $q$ boundary zone sensors $\left(\mathcal{C}_{k}^{\Gamma}\right)_{1 \leq k \leq q}$, where $\mathcal{C}_{k}^{\Gamma}=\left(\Gamma_{k}, f_{\Gamma_{k}}\right), \Gamma_{k} \subset \partial \Omega$ and $f_{\Gamma_{k}} \in L^{2}\left(\Gamma_{k}\right)$, the observation error function defined in (21) is denoted by $E_{\omega}\left(z_{0}^{\star}, \Gamma_{1}, \Gamma_{2}, \ldots, \Gamma_{q}\right)$ and depends on to the number of sensors. Similarly to the case of internal zone sensors, we can prove the following result.

Proposition 12. If the number of boundary zone sensors increases, the observation error $E_{\omega}\left(z_{0}^{\star}, \Gamma_{1}, \Gamma_{2}, \ldots, \Gamma_{k}\right)$ decreases.

When the sensors are pointwise and located on the boundary $\partial \Omega$, we denote by $\left(b_{i}^{\Gamma}, \delta_{b_{i}}\right)$ the boundary pointwise sensor where $b_{i}^{\Gamma} \in \partial \Omega$ for $1 \leq i \leq q$ and assume that they are $\omega$-strategic.

For $1 \leq i \leq q$ we consider the location $b_{i}^{\Gamma}$ of the $i$-th sensor. Assume that

$$
\left\{b_{i}^{\Gamma}\right\}=\bigcap_{k \geq 1} \Gamma_{i}^{k},
$$

where the closed sets $\Gamma_{i}^{k} \subset \partial \Omega$ satisfy $\Gamma_{i}^{k+1} \subset \Gamma_{i}^{k}$ and can be considered as the supports of boundary zone sensors satisfying, for each $k$,

$$
\mathcal{C}_{k+1, i}^{\Gamma} \leq \mathcal{C}_{k, i}^{\Gamma} .
$$

This means that $\mathcal{C}_{k, i}^{\Gamma}$ extends $\mathcal{C}_{k+1, i}^{\Gamma}$, for each $i$. The sensor supports $\left(\Gamma_{i}^{k}\right)$ are spatially shrinking and converging to $b_{i}^{\Gamma}$ as $k \longrightarrow \infty$. Thus we have the following result.

Proposition 13. For each $i, 1 \leq i \leq q$, let $E_{\omega}\left(z_{0}^{\star}, b_{i}^{\Gamma}\right)$ denote the observation error related to the boundary pointwise sensor $\left(b_{i}^{\Gamma}, \delta_{b_{i}}\right)$, and $E_{\omega}\left(z_{0}^{\star}, \mathcal{C}_{k, i}^{\Gamma}\right)$ denote the observation error related to the boundary zone sensors $\left(\mathcal{C}_{k, i}^{\Gamma}\right)$ assumed to satisfy (58). Then we have

$$
E_{\omega}\left(z_{0}^{\star}, b_{i}^{\Gamma}\right)=\lim _{k \rightarrow+\infty} E_{\omega}\left(z_{0}^{\star}, \mathcal{C}_{k, i}^{\Gamma}\right) .
$$

The above results can be proved in the same manner as in the internal case because no additional assumption on the state regularity is needed. Finally, the obtained results show that, in all cases, by increasing the number of sensors or the sensor support area we can improve the quality of the observations.

\section{Conclusion}

We have studied the relation between the observation error function and the structure and number of sensors for general distributed parameter systems. The space variable allows us to explore the structure and number of sensors in both internal and boundary cases. By introducing the notion of sensor extension, we proved an intuitive result which stipulates that when the support of an $\omega$-strategic sensor is widened, the observation error decreases. The results were demonstrated for general classes of distributed systems, and since the 1980s these results have been numerically tested on various examples but never rigorously proved. The case where the number of sensors increases by considering different combinations of sensors will be inrestigated in a future paper.

\section{Acknowledgment}

This work was carried out with the help of the Academy Hassan II of Sciences and Engineering.

\section{References}

Afifi, L., El Jai, A. and Zerrik, E. (2008). Analyse régionale des systèmes distribués linéaires, Presses Universitaires de Perpignan, Perpignan.

Amouroux, M., El Jai, A. and Zerrik, E. (1994). Regional observability of distributed systems, International Journal of Systems Science 25(2): 301-313.

Curtain, R. F. and Pritchard, A. J. (1978). Infinite Dimensional Linear Systems Theory, Springer, Berlin. 
El Jai, A. (1977). Sur la commande avec estimation de l'état initial d'une classe de systèmes distribués, Rairo Systems Analysis 11(4): 3-34.

El Jai, A. and El Yacoubi, S. (1993). On the number of actuators in parabolic systems, International Journal of Applied Mathematics and Computer Science 3(4): 673-686.

El Jai, A. and Hamzaoui, H. (2008). On actuators number in distributed systems, Sensors and Actuators (SNA6267) (A 147): 273-278.

El Jai, A. and Najem, A. (1984). Optimal actuator location in a diffusion process, Lectures Notes in Control and Information Science 62, Springer-Verlag, Berlin.

El Jai, A. and Pritchard, A. J. (1988). Sensors and actuators in distributed systems analysis, Ellis Horwood Series in Applied Mathematics, J.Wiley \& Sons, New York, NY.

El Jai, A., Pritchard, A.J., Simon, M.C. and Zerrik, E. (1995). Regional controllability of distributed parameter system, International Journal of Control 62(6): 1351-1365.

El Jai, A., Zerrik, E.H., Simon, M.C. and Amouroux, M. (1995). Regional observability of a thermal process, IEEE Transactions on Automatic Control 40(3): 518-521.

Korbicz, J. and Gawłowicz, P. (1990). Sensors location problem for stochastic non-linear discrete-time distributed parameter systems, Control of Distributed Parameter Systems, IFAC Symposia Series, Vol. 3, Pergamon Press, London, pp. 479-484.
Micheletti, A. M. (1976). Perturbazione dello spettro di un operatore ellitico di tipo variazionale, in relazione ad una variazione del campo, Ricerche di matematica. Fasc. II 25(Fasc. II).

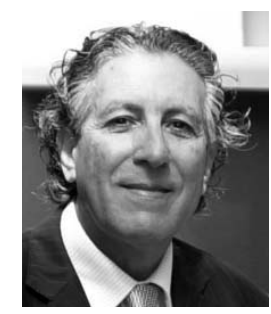

Abdelhaq El Jai is a full professor at the University of Perpignan. His research activity over the thirty past years has been focused on various aspects of the modeling, analysis and control of distributed parameter systems. $\mathrm{He}$ is a permanent member of the Academy Hassan II of Science and Technology.

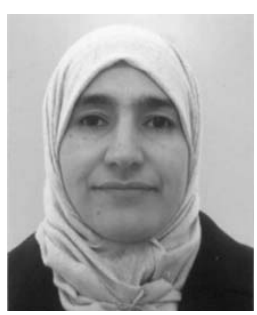

Houria Hamzaoui has been a full time researcher at the University of Perpignan for many years. Her Ph.D. was obtained at the University of Perpignan in 2008. Her research interest is focused on the relations between inputs/outputs and the analysis of distributed parameter systems.
Received: 31 January 2008 Revised: 23 June 2008 